\title{
Thermal Equilibration in the Cavity Volume of a Farmer Ion Chamber for Routine Dosimetry
}

\author{
Yuichi Kato1,3, Hiraku Fuse' ${ }^{2}$, Kazuya Shinoda',3, Katsumi Miyamoto ${ }^{3}$, Tatsuya Fujisaki ${ }^{2}$ \\ ${ }^{1}$ Graduate School of Radiological Sciences, Ibaraki Prefectural University of Health Sciences, Ami Town, Japan \\ ${ }^{2}$ Department of Radiological Sciences, Ibaraki Prefectural University of Health Sciences, Ami Town, Japan \\ ${ }^{3}$ Department of Radiological Technology, Tsukuba Medical Center Hospital, Tsukuba City, Japan \\ Email: ykato0906@yahoo.co.jp
}

Received 24 September 2015; accepted 1 November 2015; published 4 November 2015

Copyright (C) 2015 by authors and Scientific Research Publishing Inc.

This work is licensed under the Creative Commons Attribution International License (CC BY). http://creativecommons.org/licenses/by/4.0/

(c) () Open Access

\begin{abstract}
A Farmer ion chamber with an air cavity volume is the most widely used dosimeter for accurate dose determinations in radiotherapy. The quantity of ionization in the cavity volume occurred a given radiation dose has to be corrected to the cavity air temperature according to a dosimetry protocol because the mass of air in the cavity volume is subject to atmospheric variations. In the present study, we aim to measure the thermal equilibration time in the cavity volume of a Farmer ion chamber for the routine dosimetry. The Farmer ion chamber's electrode was replaced by a thin thermocouple and coated by the PMMA for a waterproofing so that the measurement of the temperature in the cavity performed in water. As a result of the measurement, A Farmer ion chamber in thermal equilibrium with waterproofing equilibrates rapidly, followed by an exponential fall-off. In water, equilibration to less than $10 \%$ of the initial temperature difference required only a few minutes. Thermal equilibrium time is hardly affected by the room temperature change.
\end{abstract}

\section{Keywords}

Farmer Ion Chamber, Thermal Equilibrium Time, Temperature Characteristic, Routine Dosimetry

\section{Introduction}

A Farmer ion chamber with an air cavity volume is the most widely used dosimeter for accurate dose determinations in radiotherapy. The quantity of ionization in the cavity volume from a given radiation dose has to be cor-

How to cite this paper: Kato, Y., Fuse, H., Shinoda, K., Miyamoto, K. and Fujisaki, T. (2015) Thermal Equilibration in the Cavity Volume of a Farmer Ion Chamber for Routine Dosimetry. International Journal of Medical Physics, Clinical Engineering and Radiation Oncology, 4, 268-272. http://dx.doi.org/10.4236/ijmpcero.2015.44032 
rected to the cavity air temperature according to a dosimetry protocol because the air mass in the cavity volume is subject to atmospheric variations [1]-[3]. However, there is limited data on the thermal equilibrium in the cavity volume of a Farmer ion chamber using routine dosimetry. In the practice of reference dosimetry in a water phantom for clinical high-energy photon beams, significant errors may occur by assuming that the cavity air temperature is the same as that of the surrounding water or the ambient air. The temperature in a Farmer ion chamber may be significantly different from the temperature measured by a wall thermometer or in the water phantom, even if the Farmer ion chamber has been acclimated in the same room for several hours. Greater temperature differences may also exist between the air in a storage room and that in the treatment room due to different room temperature settings. Several reports provided equilibration data for various Farmer ion chambers and a parallel-plate chamber in air, in water and in water equivalent plastic phantoms [4]-[7]. These reports determined time zero as that when the chamber was brought from the outside into the measurement room. It was suggested that the cavity volume of a Farmer ion chamber and the ambient air in a measurement room did not establish thermal equilibrium. In contrast, we determined time zero as that when the cavity volume of a Farmer ion chamber and the ambient air in the measurement room achieved thermal equilibrium. Routine dosimetry in Japan mostly use a PTW 30013 Farmer model, we focused on this chamber type. The central electrode is made of aluminium of $1.1-\mathrm{mm}$ diameter, and the wall materials have an outside coating of $0.335-\mathrm{mm}$ polymethyl methacrylate (PMMA) and an inside coating of $0.09-\mathrm{mm}$ graphite. The aim of the present study was to measure the thermal equilibrium time in the cavity volume of a Farmer ion chamber in routine dosimetry when the cavity volume of the chamber and the ambient air in a measurement room achieved thermal equilibrium. The central electrode of a Farmer ion chamber was replaced with a thermocouple and outside coated with PMMA on the outside for waterproofing so that the measurement of the temperature in the cavity could be performed in water. Environment of measuring the thermal equilibrium time was assumed that the temperature of the water phantom is different from the cavity air or the ambient air.

\section{Materials and Methods}

The chamber used in this study was a PTW 30013 Farmer type (PTW Freiburg GmbH, Freiburg, Germany) that was coated with PMMA for waterproofing. The central electrode in the Farmer ion chamber was replaced with a fluorocarbon resin capsule K-type thermocouple (Raythermo Industry Co., Ltd., Saitama, Japan) that provides a fast temperature response to directly measure the temperature in the Farmer ion chamber cavity. The thermocouple had a diameter of $1.1 \mathrm{~mm}$, which was equal to that of the central electrode. The thermocouple cable outside the stem was wrapped in a polyethylene tube to insulate the cavity air from ambient air, and central electrode was then replaced with the thermocouple. As shown in Figure 1, the central electrode for measuring the temperature in the cavity volume of a Farmer ion chamber was replaced with a central electrode and cable. The structure of the chamber was similar to that of PTW 30013 Farmer type chamber to vent the air through the cable. The thermometer was a thermo-logger AM-8000K (Anritsu Meter Co., Ltd., Tokyo, Japan). Temperature was recorded in degrees centigrade, as this scale provided higher resolution $\left(0.1^{\circ} \mathrm{C}\right)$ and greater accuracy $( \pm 0.1 \%$ of reading $+1^{\circ} \mathrm{C}$ ). The high-speed mode is capable of obtaining measurements at 100 -ms intervals. To assess the

PTW 30013 Farmer type chamber

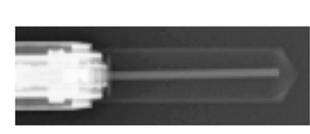

X-ray images

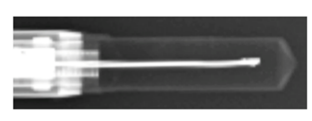

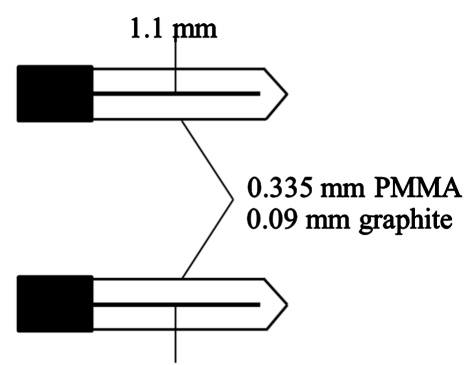

$1.1 \mathrm{~mm}$ Thermocouple

Figure 1. Farmer ion chamber X-ray images and dimensions for measuring the temperature in the cavity volume. The thermocouple was replaced with a central electrode and cable. The central electrode of a PTW 30013 Farmer type chamber is made of aluminium. The chamber used in this experiment was constructed of a nickel-chromium alloy. 
time required for thermal equilibrium in a Farmer ion chamber with a thermocouple in water, the water temperature was set at $20^{\circ} \mathrm{C}-30^{\circ} \mathrm{C}$ warmer or cooler than the surrounding room-air temperature of $22^{\circ} \mathrm{C}$ or $25^{\circ} \mathrm{C}$. The chamber temperature was equilibrated to room temperature, and only the water temperature was different from the chamber and the room temperature. The chamber was placed in water at a depth of $10 \mathrm{~cm}$ as the calibration depth [1]. The time at which the chamber was placed in the water was considered as time zero. The chamber temperature was measured to reach the equilibrium in the water temperature. For all measurements, the read-out of elapsed time and the thermocouple were simultaneously recorded with a thermo-logger. All measurements were repeated three times at each water temperature and the mean value was calculated from the measured values.

\section{Results and Discussion}

The time after time zero is symbolized as t, the temperature difference between the chamber and water as $\Delta T$, the initial temperature difference as $\Delta T_{0}$ and the initial water temperature as $T_{0}$. Typical cooling and warming plots at a room air temperature of $22^{\circ} \mathrm{C}$ are presented in Figure 2. Similarly, plots at a room air temperature of $25^{\circ} \mathrm{C}$ are presented in Figure 3. The coefficient of variation for three measurements at each water temperature maximum value was 0.02 . Tailor et al. noted that the initial shoulder in a plot represented a delay in heat transmission through the 4.6-mm Lucite wall of the build-up cap [8]. The wall thickness and materials of the chamber were $0.335 \mathrm{~mm}$ of PMMA and $0.09 \mathrm{~mm}$ of graphite. No delay was reported in heat transmission through the wall for approximately one-tenth of its thickness.

The thermal equilibration time of the chamber in a water phantom is shown in Figure 4. The squares and triangles represent cooling and warming data, respectively.

The two sets of data corresponding to warming and cooling are in good agreement and within measurement error. The equilibrium times were similar to the elapsed time during the warming and cooling phases. The equilibrium time during the warming and cooling phases at room temperatures of $22^{\circ} \mathrm{C}$ and $25^{\circ} \mathrm{C}$ were strongly correlated $(P<0.01)$, indicating that the cavity temperature is only slightly affected by differences in the room temperature and the measurement conditions during the warming and cooling phases. The characteristics of rapid equilibration and single exponential components are typical of a waterproofed chamber in water. Since in routine dosimetry the dosimeter is preset to treatment mode, the radiation measurements could begin within a few minutes from time zero. Although the two sets of data showed reasonable scatter patterns with some overlap

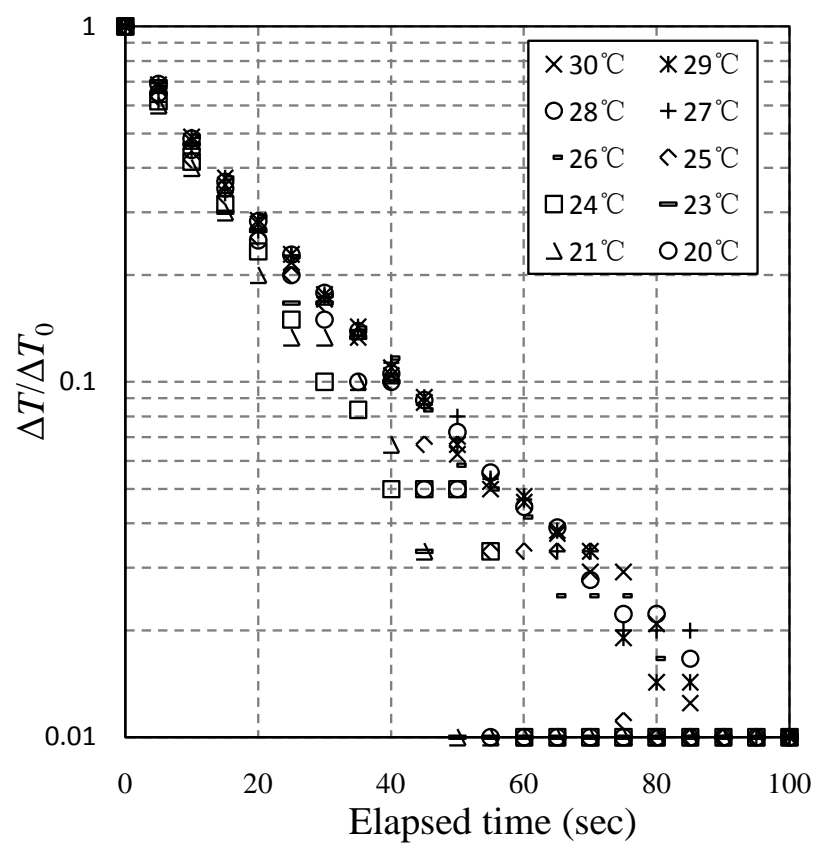

Figure 2. Plots at a room air temperature of $22^{\circ} \mathrm{C}$. The data set from $20^{\circ} \mathrm{C}$ to $30^{\circ} \mathrm{C}$ was typical for a chamber cavity warmed to these temperatures. 


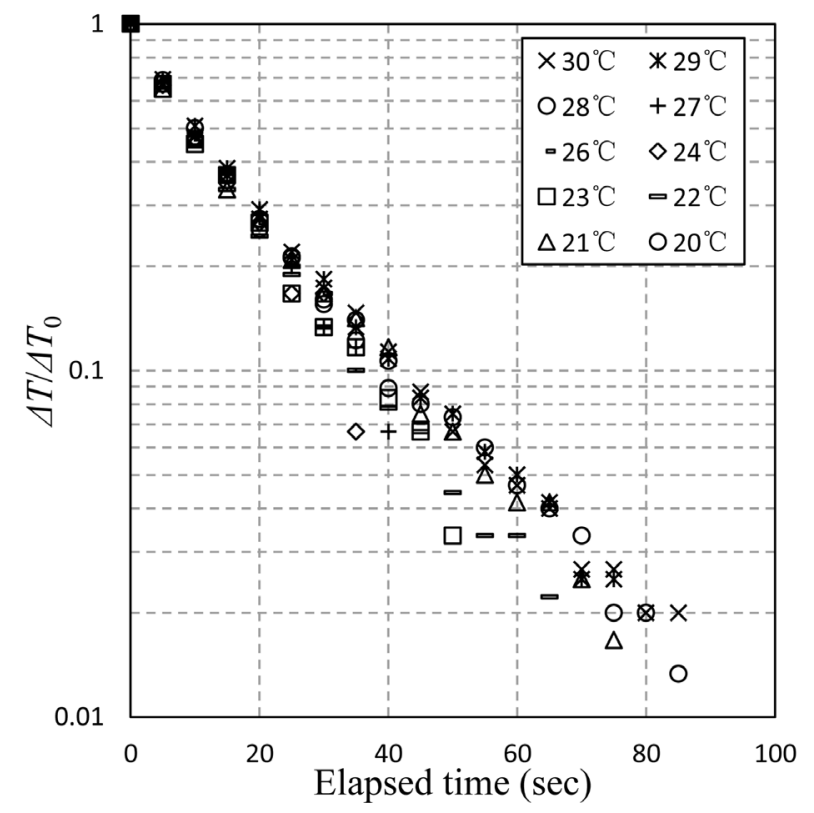

Figure 3. Plots at a room air temperature of $25^{\circ} \mathrm{C}$. The data set from $20^{\circ} \mathrm{C}$ to $30^{\circ} \mathrm{C}$ was typical for a chamber cavity warmed to these temperatures.

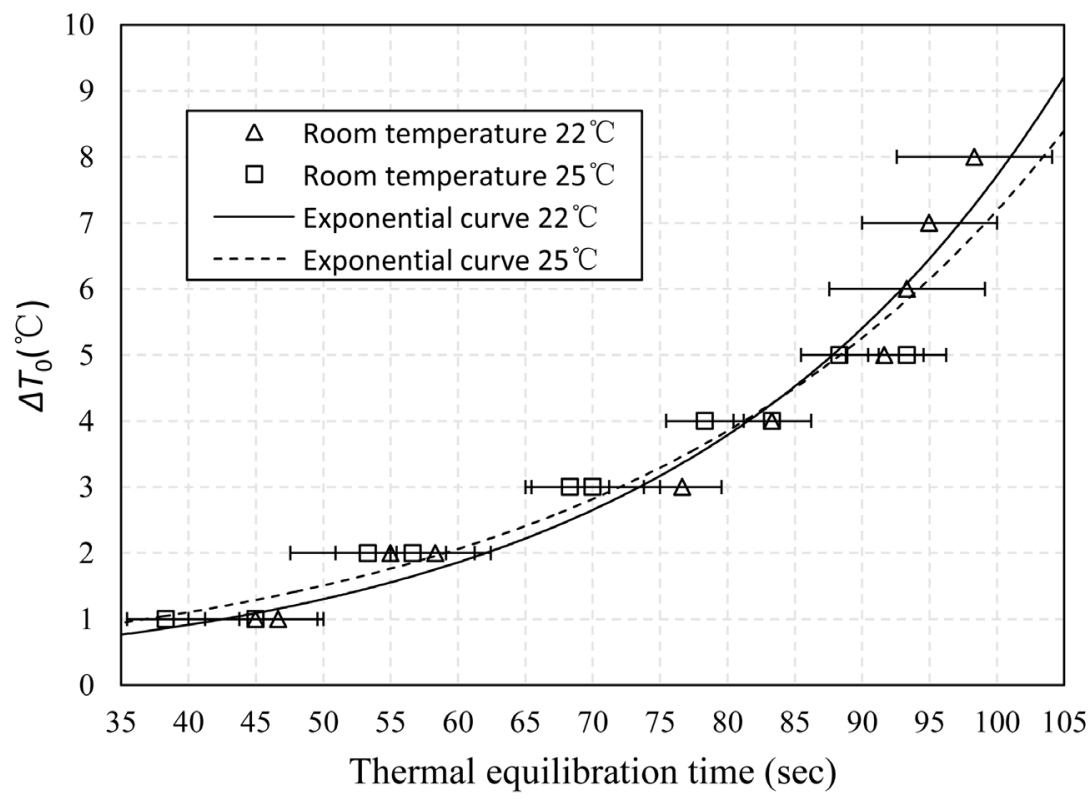

Figure 4. The squares and triangles represent the thermal equilibrium time of cooling and warming data at room air temperatures of $22^{\circ} \mathrm{C}$ and $25^{\circ} \mathrm{C}$, respectively.

and crossover, the difference may represent the time lag between the temperature change and the change in the mass of the gas inside the cavity volume. If so, this indicates a time lag of a fraction of a minute. The results of thermal equilibration of an ion chamber when introduced into water were summarized by Tailor et al. [8]. A bare thimble equilibrates very rapidly (approximately $80 \mathrm{~s}$ ) in water with virtually no shoulder. In this study, the time interval for equilibration to $10 \%$ of the initial temperature difference was up to $40 \mathrm{~s}$ in water. Tailor et al. also noted that the second exponential component was probably attributable to the influence of the stem [8]. Further, the experimental data of a bare thimble were obtained by measurement of a Farmer ion chamber into a build-up cap already at thermal equilibrium in the water phantom. 


\section{Conclusion}

Here, we present thermal equilibration data of a Farmer ion chamber coated with PMMA for waterproofing without the build-up cap. A Farmer ion chamber in thermal equilibrium with waterproofing equilibrates rapidly, followed by an exponential fall-off. In water, equilibration to less than $10 \%$ of the initial temperature difference required only a few minutes. Thermal equilibrium time is hardly affected by the room temperature change.

\section{References}

[1] Japan Society of Medical Physics (2012) Standard Dosimetry of Absorbed Dose to Water in External Beam Radiotherapy 12. Tsusho-Sangyo Kenkyu-Sha, Tokyo.

[2] International Atomic Energy Agency (2000) Technical Reports Series No. 398, Absorbed Dose Determination in External Beam Radiotherapy. International Atomic Energy Agency, Vienna.

[3] Almond, P.R., Biggs, P.J., Coursey, B.M., Hanson, W.F., Saiful Huq, M., Nath, R. and Rogers, D.W.O. (1999) AAPM Task Group No. 51, Protocol for Clinical Reference Dosimetry of High-Energy Photon and Electron Beams. Medical Physics, 26, 1847-1870. http://dx.doi.org/10.1118/1.598691

[4] Hiraoka, T., Kawashima, K., Hoshino, K. and Matsuzawa, H. (1982) Temperature and Pressure Characteristics of JAPM Dosimeter and Long-Term Stability of Several Barometers. Nippon Acta Radiologica, 42, 1137-1146. (In Japanese)

[5] Kubo, H. (1983) Ionization Chamber Response to a Sudden Change of Ambient Temperature in Air, Water and a Polystyrene Phantom. Medical Physics, 10, 676-679. http://dx.doi.org/10.1118/1.595363

[6] van der Giessen, P.H. (1986) About the Rate of Temperature Changes in a Thimble Chamber. Radiation Oncology, 7, 287-291. http://dx.doi.org/10.1016/S0167-8140(86)80040-8

[7] Mayo, C.S. and Gottschalk, B. (1992) Temperature Coefficient of Open Thimble Chambers. Physics in Medicine and Biology, 37, 289-291. http://dx.doi.org/10.1088/0031-9155/37/1/022

[8] Tailor, R.C., Chu, C., Followill, D.S. and Hanson, W.F. (1998) Equilibration of Air Temperature Inside the Thimble of a Farmer-Type Ion Chamber. Medical Physics, 25, 496-502. http://dx.doi.org/10.1118/1.598226 